\title{
EXTENDING THE FATIGUE LIFE OF RIVETED BRIDGES USING DATA FROM LONG TERM MONITORING
}

\author{
Eugen Brühwiler \\ Civil Engineering Institute, Swiss Federal Institute of Technology (EPFL), \\ Station 18, CH-1015 Lausanne, Switzerland \\ (Email: eugen.bruehwiler@epfl.ch)
}

\begin{abstract}
A methodology inherent to existing structures is presented for the fatigue safety verification of riveted bridges. The suggested approach allows for the determination of updated action effects explicitly considering data from long term monitoring. Data from monitoring allow for accurate determination of fatigue relevant stresses in fatigue prone bridge elements, and uncertainties in the determination of updated action effects are reduced. By means of the presented approach, the fatigue safety of a riveted railway bridge of high cultural heritage value was verified after 115 years of service duration. Data from monitoring were exploited by Rainflow analysis and served as the basis for the fatigue safety verification. As the locations of measurements are generally not identical with the cross sections of verification, measured strains were translated to the relevant verification cross section by means of factors that were determined by structural analysis. Sufficient fatigue safety was finally verified for the entire riveted structure and additional service duration of at least 50 years was validated.
\end{abstract}

Keywords: Fatigue safety, Service life, Riveted steel bridge, Fatigue damage accumulation, Structural health monitoring, Examination

DOI: $10.18057 /$ IJASC.2015.11.3.3

\section{INTRODUCTION}

In many countries, civil structures have been in service already for several generations. As part of the transportation infrastructure, bridges add value to the public economy. Therefore, there is high interest in economic performance while providing unrestricted utilisation (e.g. without limits on traffic loads) and responding to increasing traffic demands. Obviously, there is a need to extend the service life of civil structures even further, i.e., significantly beyond 100 years, which often is the arbitrarily presumed service life of structures.

In this context, structural engineers have to devise novel ways to examine the structural safety of existing structures, in particular when high cultural values are involved. The contemporary approach is based on an inherent methodology that essentially includes collecting detailed in situ information about the structure, for example, by long term monitoring of structural behaviour. Parameters controlling structural safety are determined more precisely and, for example, the structural safety of an existing bridge can be proved using so-called updated values for actions (loads) and resistance. In this way, it can often be shown that an existing structure may be subjected to higher load effects while meeting the safety requirements, thereby avoiding intervention.

Regarding existing bridges, a greater source of uncertainty lies on the traffic loading or more specifically the action effects arriving in the structural elements. In recent years, increasingly sophisticated approaches have emerged for load effect estimation using traffic simulations incorporating Weigh-in-Motion (WIM) data which form the basis of load models in design and "assessment" codes. While vital for design, these codes are based on generic heavy vehicle data from a range of locations and therefore may not always represent the site conditions at the existing bridge under investigation. In addition, codes include provisions for illegally overloaded vehicles 
which can be reduced substantially through adequate policing (on roads and also on railways) which varies hugely between countries.

An important distinction between existing bridge safety verification and fatigue design of a new bridge is that an existing bridge structure can be monitored to determine the real 'action effects' experienced. The advent of cheap and high storage capacity hardware in recent years means that direct measurement of elemental action effects via structural monitoring is now a viable option. Monitoring can overcome limitations of accurately modelling in-service behaviour at an elemental level. For example, material properties may change over time and secondary elements may reduce the stress levels in the structural parts which can provide uncertainty in modelling but these effects are inherent in the measured data. In addition, reliable local WIM data is not always available.

This paper introduces a novel approach to verify and predict the future service duration and the fatigue safety of bridges using data from monitoring. The approach is illustrated by means of an application case in Switzerland related to a larger riveted railway bridge of high cultural value and in service for more than 115 years. It will be shown that there are no 'old' bridges, but bridges that provide adequate performance (or not). Extending the service life of bridges by following the approach presented in this paper may allow continuous utilisation of existing structures rather than their replacement. This approach is clearly in agreement with the principles of sustainable development.

\section{PROPOSED APPROACH}

\section{$2.1 \quad$ Introduction}

Examination (also called "assessment") of an existing structure may be performed following the principles such as the ones defined in the Swiss Standards for existing structures enforced by the Swiss Society of Engineers and Architects (SIA) (Brühwiler et al. [1], SIA 269 [2]). In Switzerland, these standards provide the regulative basis the structural engineer can rely on to deal professionally with existing structures.

These principles lead to a methodology inherent to existing structures which has already been successfully applied over the last 25 years. Yet, many structural engineers nowadays still apply codes for the design of new structures to assess existing structures. This is fundamentally wrong and this over-conservative approach often leads to unnecessary and costly interventions. A change of paradigm is urgently needed aiming the structural engineering community to clearly distinguish between codes for new structures and codes for existing structures.

The contemporary approach to existing structures is based on updating, which means collecting and exploiting detailed in-situ information from the existing structure while reducing uncertainties in structural parameters. The controlling parameters are determined as precisely as needed following a stepwise procedure with increasing focus on details. The general examination comprises the whole structure with the objective to identify aspects that need to be examined in more detail. One or more detailed examinations follow with the focus on the identified aspects.

\subsection{Structural Safety Verification Format}

The structural safety verifications are performed using updated values, called examination values, with the objective to verify for the existing structure that the relevant limit states are not exceeded. Usually, deterministic verification is conducted, and the notion of degree of compliance $n$ is introduced in the deterministic verification of structural safety: 
$n=\frac{R_{d, \text { updated }}}{E_{d, \text { updated }}}$

where $R_{\text {d,updated }}$ and $E_{\text {d,updated }}$ are the examination values of resistance and action effect, respectively. The degree of compliance is a numerical statement showing the extent to which an existing structure fulfils the structural safety requirements. This formulation not only gives the information whether the structural safety is fulfilled, i.e. $n \geq 1,0$, it also indicates by how much the verification is fulfilled (or not). The latter is necessary for the evaluation of results and in view of the planning of interventions.

In this context, monitoring of structural behaviour has thereby the general objective to determine more accurately effective action effects in terms of measured strains (stresses), displacement or accelerations, arriving in structural elements such as relevant parts of steel structures, steel reinforcing bars and concrete in reinforced concrete or other structures.

\subsection{Determination of Updated Action Effect}

In the first step of verification on Level 1 (general examination), the examination value of action effect $E_{\text {d,updated }}$ is determined using updated load models (for permanent and live loads) (SIA 269/1 [3]) which are applied to a structural model to obtain sectional forces, stresses and strains, i.e. action effects, through structural analysis (Figure 1). This approach is analogous to the one generally applied for the design of new structures. While this simple approach may lead in many cases to suitable results (i.e. showing that structural safety is fulfilled), it only takes partly advantage of the fact that the existing structure exists and thereby information may be gained.

If safety verification is not conclusive on Level 1, further updating may be performed on Level 2 in a detailed examination, by performing monitoring of action effects due to live loads (Figure 1). In this way, action effects are directly measured by monitoring reducing thus sources of uncertainty.

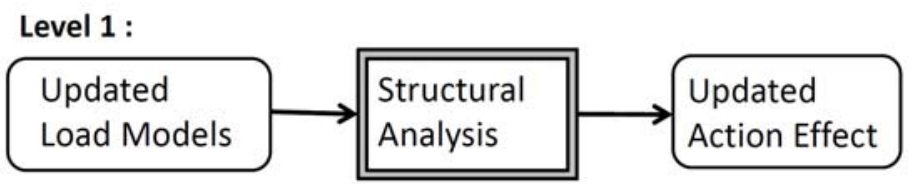

Level 2 :

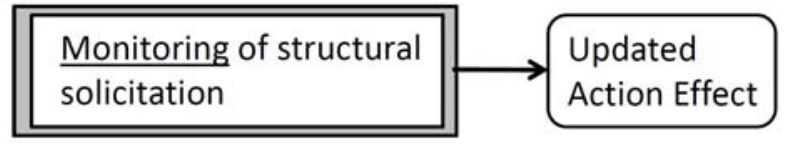

Figure 1. Determination of Updated Action Effect

However, it may not always be possible to obtain data from monitoring directly in the cross sections determinant for the safety verification. Also, action effect due to permanent load (dead load) obviously cannot be recorded by monitoring. Structural analysis is thus needed to "translate" data from monitoring to sections determinant for the structural safety verification (Figure 2) and to determine the solicitation of the existing structure due to permanent loads. 


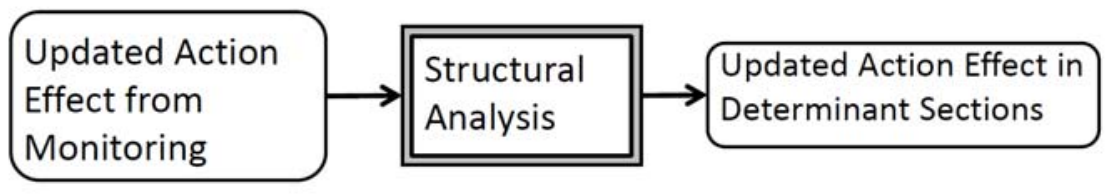

Figure 2. Translation of Data from Monitoring to Sections Determinant for Structural Safety

Consequently, the use of structural models changes its importance when compared to structural analyses for the design of new structures. Also, data from monitoring are used to calibrate the structural model with the objective to obtain sectional forces as accurate as possible thereby reducing uncertainty usually implied in structural analysis.

Such a calibrated structural model may then be used to determine, for example, fatigue action effects of future traffic scenarios. Note that following the approach on Level 2, no more load model is actually needed as solicitation of structural elements is directly recorded which obviously is the most reliable data to verify structural safety.

\subsection{Safety Requirements}

The structural safety requirements imposed on an existing structure need to be clearly defined as they may have a major influence on the extent of interventions. Monitoring of action effects on structural elements reduces uncertainties. Yet, some safety margin needs to be respected, either by considering a partial safety factor or, in the case of fatigue safety verification, by some criterion of target level of acceptable fatigue damage.

\section{CASE STUDY OF A RIVETED RAILWAY BRIDGE}

The approach presented in Chapter 2 has been applied for the verification of fatigue safety of a riveted steel railway bridge as shown in the following. More detailed information on this case study is given in Brühwiler et al. [4].

\subsection{Description of the Bridge}

The railway bridge across the River Rhine at Eglisau in Switzerland was built from 1895 to 1897 for single lane railway traffic. The central part of the $457 \mathrm{~m}$ long bridge is a riveted steel truss structure made of early mild steel. The truss has a span of $90 \mathrm{~m}$ and a height of $9 \mathrm{~m}$. Multiple arch approach viaducts in natural stone masonry with piers up to $50 \mathrm{~m}$ in height follow in the north and south of the steel structure (Figure 3).

In 1982/83, the original carriageway (that consisted of a ballasted track on Zores iron profiles) was replaced by a steel trough with ballast. This led to the situation that structural parts of the carriageway (which are usually of most fatigue relevance) are no longer determinant. In the following, only the main girder is discussed. 


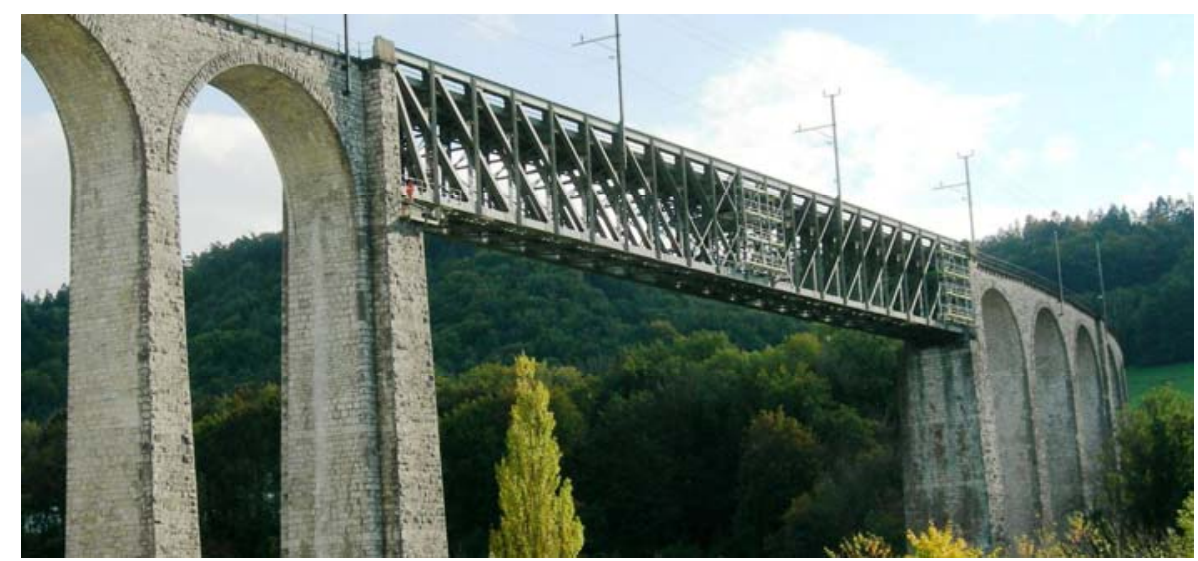

Figure 3. Railway Bridge across the River Rhine at Eglisau, Switzerland

\subsection{Model for Structural Analysis}

In the first phase of this case study, the truss structure was modelled by means of a three-dimensional bar model in order to determine sectional forces necessary for structural safety verification. A three-dimensional model was necessary because the carriageway (consisting of the trough, cross girders and floor beams) is located approximately $1.5 \mathrm{~m}$ below the top chord of the main truss and thus contributes to the global structural behaviour by carrying significant compression forces in addition to the top chord. The nodes of the main truss were modelled with fixed connections and eccentricity was taken into account. However, the bars of the upper and lower wind bracing as well as of the transverse bracings were modelled with pinned connections. Comparison of the results from the load tests and structural analysis confirmed the adequacy of the structural model allowing calculating the structural behaviour with sufficient precision.

\subsection{Monitoring}

Strain gauges relevant for the verification of the fatigue safety were mounted on structural elements and zones that are essentially subjected to tensile stresses in (Figure 4):

- Field 1 on the two tensile diagonals and posts of the main truss and on the trough, floor beams and cross girders of the carriageway, and

- Field 10 on the lower chord at mid-span.

In addition to the criterion of having good accessibility, strain gauges were placed such that strain distribution in the measured cross section corresponds most likely to gross section stresses (Figure 4 right). This means, that the measuring locations lie as far away as possible from rivet holes or other "hot spots", to avoid measuring any stress concentration close to rivets. Figure 5 (left) shows some cross sections with the arrangement of strain gauges. 

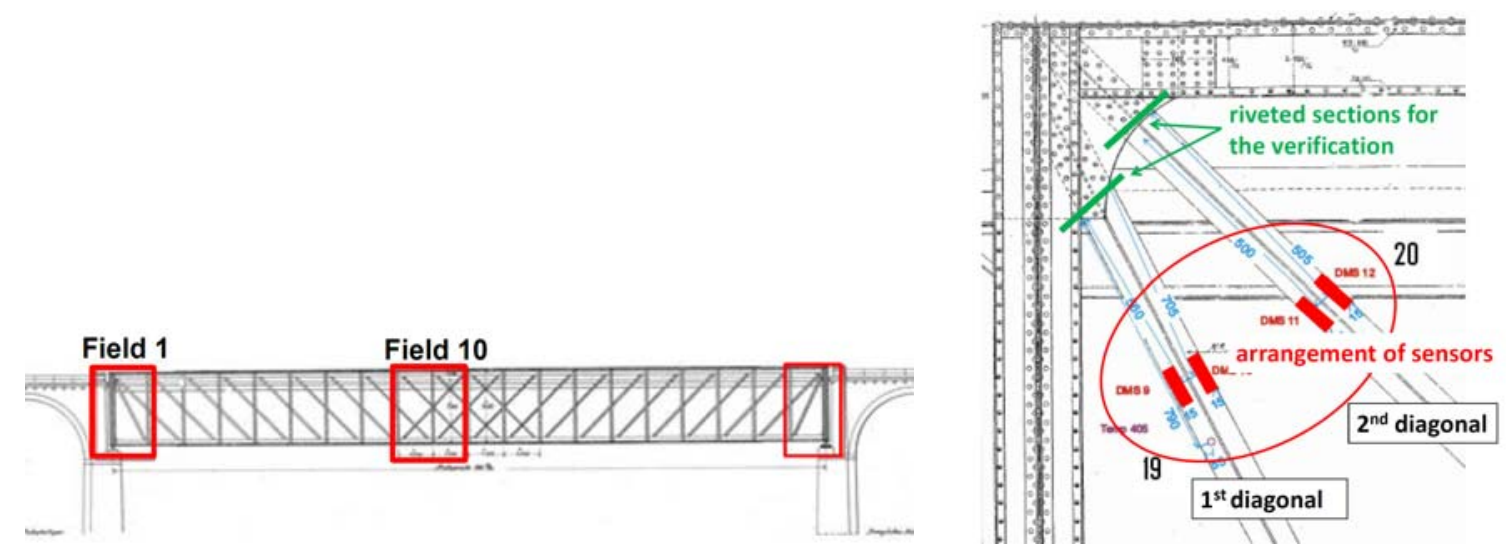

Figure 4. Left: Localisation of the Strain Gauges Relevant for the Fatigue Safety Verification; Right: Location of Sensors and Verification Sections of Diagonals in Field 1

The strain readings of all sensors due to the passage of every train, including passenger and freight trains, were recorded over a period of 12 months. As an example, Figure 5 (right) shows the structural response (as a function of time) of various elements due to the passage of a freight train. As expected, the results depict the small influence of single axle loads on diagonals and lower chord of the truss girder, while the strain readings for the cross girder show first a continuous increase (due to the global load bearing behaviour) followed by the distinct effect of the passage of each axle with rather large strain variations (local load bearing behaviour). Analysis of recorded data confirmed that the fully automatic monitoring system allowed for reliable long term readings that could subsequently be exploited as a basis for the verification of fatigue safety.
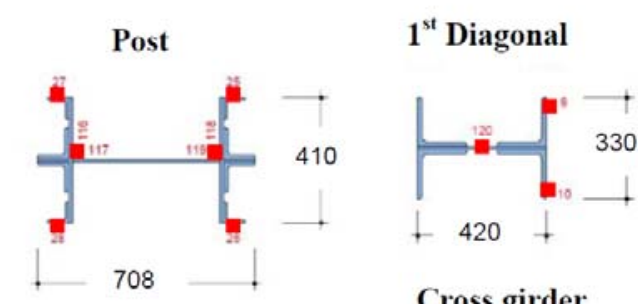

\section{Cross girder}
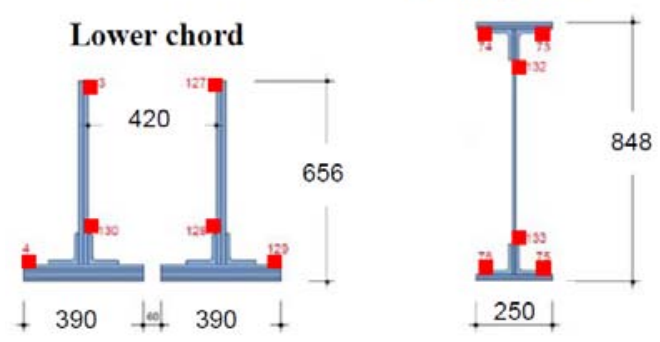
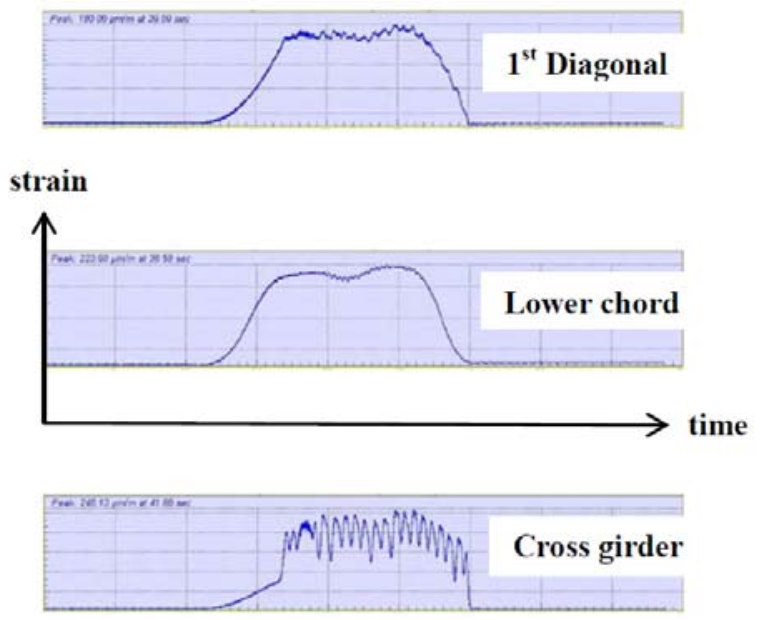

Figure 5. Left: Arrangement of Strain Gauges in Cross Sections;

Right: Recorded Data of One Gauge on the 1st Diagonal and

Lower Chord of the Truss Girder as well as on a Cross Girder

Monitored raw data from every train passage were analysed by means of Rainflow analysis, i.e. stress ranges were assigned to selected strain (stress) range groups and the number of strain ranges per group was counted. All monitored values were added over one calendar month, stored on Excel tables. Finally, the results from all 12 months of monitoring were summed up. Figure 6 shows graphically presented histograms for the two analysed diagonals (which turned out to be the most fatigue relevant elements). 


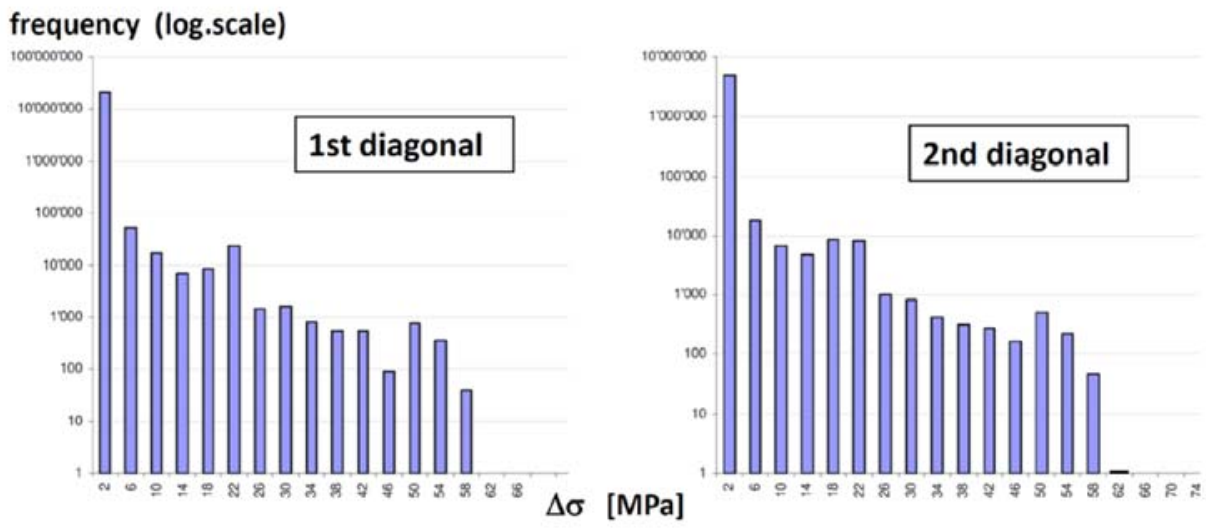

Figure 6. Histograms of a Compilation of Stress Ranges over the Whole Monitoring Period of the Two Diagonals of the Main Truss

\subsection{Fatigue Safety Verification}

Fatigue safety is verified for determinant cross sections (details) with rivets. Since the location of the strain sensors were intentionally chosen to avoid measuring any stress concentration near rivets, monitored strain (stress) values need to be translated to the determinant rivet positions of the cross sections relevant for verification (Figure 4 right). Consequently, conversion factors were determined for each verified cross section using the calibrated model for structural analysis. For this, a specific loading configuration was chosen to determine the sectional forces. Moreover, as tensile stresses obtained from monitored values are valid for gross sections, the conversion factor also considers net sectional areas in the relevant sections with rivet holes. These conversion factors were then applied to the monitored values to obtain stress range values for the fatigue safety verification.

Fatigue safety was verified with respect to fatigue resistance for riveted details as given in SIA 269/3 [5] and Taras \& Greiner [6] (Figure 7). Fatigue category of $71 \mathrm{MPa}$ (with a constant amplitude fatigue limit of $51 \mathrm{MPa}$ ) was taken into account for the diagonals and the lower chord of the main truss girder. This fatigue resistance is to be compared with stress ranges calculated using net-section stresses.

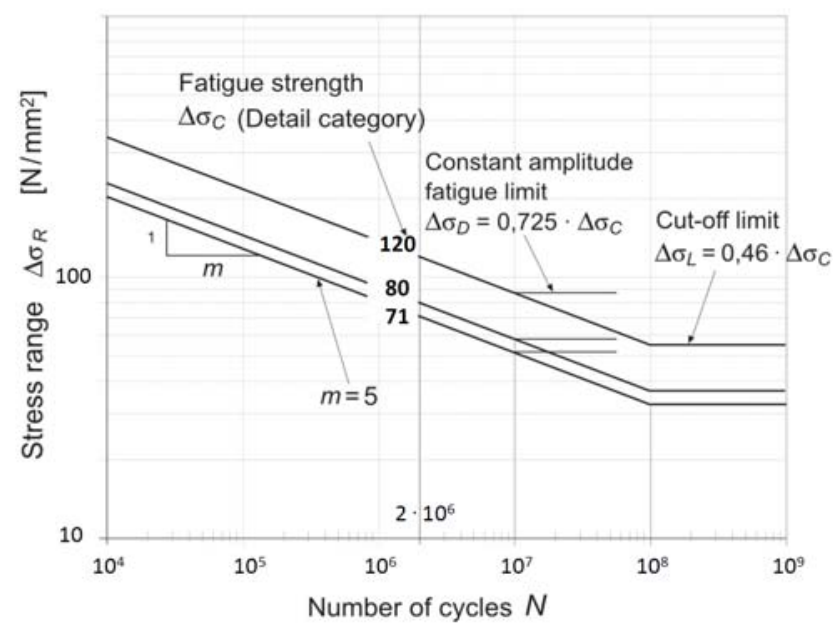

Figure 7. Fatigue Strength for Riveted Structural Details, According to SIA 269/3 [5] 
The fatigue resistance given in Figure 7 is considered to be rather conservative in particular in the domain of low stress ranges and high numbers of cycles which is the relevant domain in the present case. Indeed, constant-amplitude fatigue limit of riveted members was estimated to be at a stress range level of $70 \mathrm{MPa}$ based on fatigue tests up to 20 million cycles (Brühwiler et al. [7]); more research is needed to explore more in detail the fatigue behaviour of riveted details at high numbers of cycles.

Verification of fatigue safety followed a stepwise procedure:

- Step 1: Fatigue safety verification with respect to the fatigue limit :

For every fatigue relevant verification section, the maximum monitored value of stress range over the whole monitoring period was translated into maximum stress range for the verification using the corresponding conversion factor. The results for the two diagonals are given in Table 1. The fatigue safety is verified if the degree of conformity $n$, i.e., the ratio of fatigue limit to maximum fatigue stress range, is equal to or larger than 1.0.

Table 1. Results of the Verification with Respect to the Fatigue Limit for the Two Main Diagonals

\begin{tabular}{cccccc}
\hline $\begin{array}{c}\text { Structural } \\
\text { element }\end{array}$ & $\begin{array}{c}\text { max. stress } \\
\text { range from } \\
\text { monitoring } \\
{[\mathrm{MPa}]}\end{array}$ & $\begin{array}{c}\text { Conver- } \\
\text { sion factor }\end{array}$ & $\begin{array}{c}\text { max. stress range } \\
\text { (verific. section) } \\
\Delta \sigma_{\max } \\
{[\mathrm{MPa}]}\end{array}$ & $\begin{array}{c}\text { Fatigue } \\
\text { limit } \\
\Delta \sigma_{D} \\
{[\mathrm{MPa}]}\end{array}$ & $\begin{array}{c}\text { Degree of } \\
\text { conformity } \\
n=\frac{\Delta \sigma_{D}}{\Delta \sigma_{\max }}\end{array}$ \\
\hline $\begin{array}{c}\text { Main truss: } \\
1^{\text {st }} \text { diagonal }\end{array}$ & 58 & 1.71 & 99 & 51 & 0.52 \\
$2^{\text {nd }}$ diagonal & 62 & 1.33 & 82 & 51 & 0.62 \\
\hline
\end{tabular}

The two main diagonals do not pass the fatigue safety check with respect to the fatigue limit. Consequently, they are verified on the next verification level according to Step 2.

\section{- Step 2: Fatigue damage accumulation calculation and fatigue safety verification}

The first and second diagonals were investigated by means of Palmgren-Miner damage accumulation method using the S-N curves for riveted details (Figure 6). Stress ranges below the cut off limit were neglected.

In a first step, fatigue damage over the 12 months of monitoring (April 2011 - March 2012, average of 159 trains per day) was determined. Histograms of stress range as obtained from Rainflow analysis were translated to the verification sections by applying the conversion factors. Then, for each stress range $\Delta \sigma_{i}^{m}$, the maximum number of cycles $N_{i}$ was determined as follows:

$$
N_{i}=\frac{\Delta \sigma_{c} \cdot 2 \cdot 10^{6}}{\Delta \sigma_{i}^{m}}
$$

with $\Delta \sigma_{c}$ being the fatigue strength at 2 million cycles. The measured number of cycles $n_{i}$ for each stress range was then used to determine the theoretical fatigue damage $d_{i}$ per stress range:

$d_{i}=\frac{n_{i}}{N_{i}}$ 
And by summing up over all $k$ stress ranges, the total damage $D$ is obtained:

$$
D=\sum_{i=1}^{k} d_{i}
$$

Fatigue failure is supposed to occur if the total damage reaches a value of $D=1.0$.

Past traffic was modelled using the load models valid for the respective allowable Line Classes as defined by the European Railways (UIC Code 700 [8]). During the initial phase of railway service, traffic similar to Line Class B2 was allowed. With the electrification in 1928, traffic loads were increased to Line Class C3. With the modification of the carriageway in 1982, the Line Class was again increased to D4. In addition, reliable data was available regarding train numbers and gross register tonnage (GRT) per year from the statistical yearbooks of the Swiss Federal Railways. With this data, fatigue solicitation of the bridge during the past service period from 1897 to 2011 could be estimated with good precision. For the future railway traffic, a scenario for the expected traffic in terms of number of trains and GRT was developed in accordance with the Swiss Federal Railways and considered to forecast cumulated fatigue damage for the future. This led to the results shown in Table 2.

Table 2. Total Projected Fatigue Damage for the Diagonals

\begin{tabular}{cccc}
\hline \multirow{2}{*}{ Structural element } & \multicolumn{4}{c}{ Cumulated fatigue damage until year } \\
& 2012 & 2035 & 2065 \\
\hline $1^{\text {st }}$ diagonal & 0.133 & 0.247 & 0.420 \\
$2^{\text {nd }}$ diagonal & 0.025 & 0.045 & 0.075 \\
\hline
\end{tabular}

A maximum value of theoretical fatigue damage of 0.42 in the year 2065 was obtained for the $1^{\text {st }}$ diagonal of the main truss while for the $2^{\text {nd }}$ diagonal and the lower chord at mid-span much smaller accumulated fatigue damage was calculated. The rather large difference between the two diagonals is due to the fact that the $1^{\text {st }}$ diagonal gets higher stress values after considering the conversion factor of 1.71 (which is rather high because of significant moments due to high fixity of the $1^{\text {st }}$ diagonal) (Table 1). These higher stresses contribute over-proportionally to damage accumulation as there is a more significant number of stress range values higher than the assumed fatigue limit when compared to the $2^{\text {nd }}$ diagonal. This shows again the importance of precise stress determination and more precise knowledge of the fatigue limit. However, as all cumulated fatigue damage values are significantly lower than the theoretical value of $D=1.0$ for fatigue failure, no further detailed examination of the $1^{\text {st }}$ diagonal was performed.

No partial safety factors have hitherto been applied on the fatigue action effect and fatigue resistance values. The project team decided to accept a safety criterion given by a fatigue damage value of 0.50 as being acceptable and providing a sufficient safety margin with respect to theoretical fatigue failure of the element. The reasoning for this safety criterion comprises the good accessibility for inspection of the fatigue relevant details, the rather conservative approach in the determination of the conversion factor and the considered rather conservative fatigue category which is a 5\% fractile value established based on mean test data and which probably underestimates the fatigue endurance or high cycle domain. As the highest forecasted maximum cumulated damage value of 0.42 for a further service duration of 50 years is smaller than 0.50 , the fatigue safety verification is fulfilled for the entire riveted structure. 


\subsection{Discussion and Recommendation}

The fatigue safety verifications based on the monitored values show sufficient fatigue safety for the diagonals of the main truss and thus for the entire riveted structure for at least the next 50 years of service duration. Also, the structural elements having priority during inspections were identified.

As the determinant structural element, i.e. the $1^{\text {st }}$ tension diagonal of the main truss, showed the by far highest theoretical fatigue damage value, it is recommended to strengthen this structural element in the near future. By performing this intervention which has a preventive character and is not costly, very long future service duration (going well beyond 50 years) could be expected and attributed to the entire riveted structure, while taking into account the significantly more intense fatigue solicitation due to increasing traffic demand in the future.

Moreover, information and data regarding the railway traffic and the calculated fatigue damage (Table 2) actually indicate that the riveted structure was exposed to a relatively low fatigue solicitation during its past service duration of 115 years. In fact, the structure is today virtually still in an undamaged condition in terms of fatigue, and only the future higher railway traffic loading may produce some fatigue damage in certain structural elements.

A 115 year old bridge is commonly considered to be "old" and a thus often condemned to be a candidate for replacement. The present example shows however again, that a change in paradigm is needed when considering the notion of service duration: there is no "old" bridge, and solely the structural performance (and not the age of a structure) is the relevant and adequate criterion.

\section{CONCLUSIONS}

A methodology inherent to existing structures is presented for the fatigue safety verification leading to the following conclusions:

(1) The suggested approach to determine updated action effects allows for explicit consideration of data from long term monitoring.

(2) Data from monitoring allow for accurate determination of fatigue relevant stresses in fatigue prone bridge structures. Hence, uncertainties (due to load models and structural analysis) in the determination of updated action effects are reduced.

(3) By means of the presented approach, the fatigue safety of a riveted railway bridge of high cultural heritage value was verified after 115 years of service duration. Long future service duration may be expected taking into account higher future traffic loading.

The present approach is economic as the cost for the long term monitoring and accompanying theoretical studies is only a small fraction of the cost of a hypothetical major strengthening or bridge replacement project which could thus be avoided by this study.

\section{OUTLOOK}

Obviously, this approach may also be applied for the structural safety verification at ultimate limit state. For example, in an accompanying monitoring project of a 50 year old post-tensioned concrete highway bridge subjected to heavy traffic, over 350 days of element level continuous 
measurements have been performed to provide a unique insight into the behaviour in the monitored deck slab at an elemental level [9]. Bridge action effect estimation was approached from two separate directions, namely monitoring and simulation. The work represents a development towards improved methodologies for safety assessment based on data from monitoring. Direct measurement of strain in steel reinforcing bars has considerable potential as a means to determine the characteristic behaviour of extreme traffic action effects in bridge deck slabs. It was found that the measurement duration required to capture the extreme behaviour in reinforced concrete bridge elements is highly dependent on element location and orientation. The tested extrapolation techniques show high instability of extreme predictions for low measurement periods. Data from monitoring showed that the ULS strain and hence stress predictions are far too low to provide any fatigue or ULS concerns in this case. Hence the lifetime of many such bridge details could be extended well into the future. In some cases this new level of information could potentially be used to justify increases in legal loading without any safety risk to the users.

\section{ACKNOWLEDGMENTS}

The author wishes to thank Max Bosshard and Pirmin Steck (Flückiger + Bosshard Consulting Engineers Zurich), Christian Meyer (Terra Monitoring Zurich), Dr Marcel Tschumi and Dr Senta Haldimann (SBB Berne), all from the Eglisau Bridge project team, for their contributions to the case study and the Swiss Federal Railways (SBB) for funding.

\section{REFERENCES}

[1] Brühwiler, E., Vogel, T., Lang, T., Lüchinger, P., "Swiss Standards for Existing Structures", Structural Engineering International, IABSE Zurich, 2012, Vol. 22, No. 2, pp. 275-280.

[2] SIA 269, "Existing Structures - Bases for Examination and Interventions", Swiss Society of Engineers and Architects (SIA), Zurich, 2011.

[3] SIA 269/1, "Existing Structures - Actions", Swiss Society of Engineers and Architects (SIA), Zurich, 2011.

[4] Brühwiler, E., Bosshard, M., Steck, P., Meyer, C., Tschumi, M. and Haldimann, S., "Fatigue Safety Examination of a Riveted Railway Bridge using Data from Long Term Monitoring", Proceedings, IABSE Conference on Assessment, Upgrading and Refurbishment of Infrastructures, Rotterdam, The Netherlands, May 6 - 8, 2013.

[5] SIA 269/3, "Existing Structures - Steel Structures", Swiss Society of Engineers and Architects (SIA), Zurich, 2011.

[6] Taras, A. and Greiner, R., "Development and Application of a Fatigue Class Catalogue for Riveted Bridge Components", Structural Engineering International, IABSE Zurich, 2010, Vol. 20, No. 1, pp. 91- 103.

[7] Brühwiler, E., Smith, I. and Hirt, M., "Fatigue and Fracture of Riveted Bridge Members" , ASCE J. Struct. Eng., 1990, Vol. 116, No. 1, pp. 198-214.

[8] UIC Code 700, "Classification of Lines and Resulting Load Limits for Wagons", International Union of Railways, 2004.

[9] Treacy, M. and Brühwiler, E., "Extreme Action Effects in Reinforced Concrete Bridges from Monitoring", Proceedings, IABSE Conference on Assessment, Upgrading and Refurbishment of Infrastructures, Rotterdam, The Netherlands, May 6 - 8, 2013. 